\title{
Platelet-rich Concentrates Differentially Release Growth Factors and Induce Cell Migration In Vitro
}

\author{
Michael O. Schär MD, Jose Diaz-Romero PhD, \\ Sandro Kohl MD, Matthias A. Zumstein MD, \\ Dobrila Nesic PhD
}

Published online: 18 February 2015

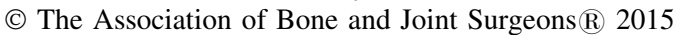

\begin{abstract}
Background Platelet-rich concentrates are used as a source of growth factors to improve the healing process. The diverse preparation protocols and the gaps in knowledge of their biological properties complicate the interpretation of clinical results.

Questions/purposes In this study we aimed to (1) analyze the concentration and kinetics of growth factors released from leukocyte- and platelet-rich fibrin (L-PRF), leukocyte- and platelet-rich plasma (L-PRP), and natural blood clot during in vitro culture; (2) investigate the migration of mesenchymal stem cells (MSCs) and human umbilical vein endothelial cells (HUVECs) as a functional response to the
\end{abstract}

One or more of the authors (MOS, MAZ, DN) have received research funding from the RMS Foundation, Bettlach, Switzerland (RMS-Nr. E11_0008) during the study period.

All ICMJE Conflict of Interest Forms for authors and Clinical Orthopaedics and Related Research ${ }^{\mathbb{R}}$ editors and board members are on file with the publication and can be viewed on request.

Clinical Orthopaedics and Related Research ${ }^{\circledR}$ neither advocates nor endorses the use of any treatment, drug, or device. Readers are encouraged to always seek additional information, including FDA-approval status, of any drug or device prior to clinical use. Each author certifies that his or her institution approved or waived approval for the human protocol for this investigation and that all investigations were conducted in conformity with ethical principles of research.

M. O. Schär, J. Diaz-Romero, D. Nesic

Department of Clinical Research, University of Bern, Bern, Switzerland

M. O. Schär, S. Kohl, M. A. Zumstein ( $ه)$

Department of Orthopaedic Surgery, Inselspital, University of

Bern, Freiburgstrasse, 3010 Bern, Switzerland

e-mail: matthias.zumstein@insel.ch factors released; and (3) uncover correlations between individual growth factors with the initial platelet/leukocyte counts or the induced cell migration.

Methods L-PRF, L-PRP, and natural blood clot prepared from 11 donors were cultured in vitro for 28 days and media supernatants collected after 8 hours and 1, 3, 7, 14, and 28 days. Released transforming growth factor $\beta 1$ (TGF- $\beta 1$ ), vascular endothelial growth factor (VEGF), insulin growth factor (IGF-1), platelet-derived growth factor AB (PDGF$\mathrm{AB}$ ), and interleukin-1 $\beta$ (IL-1 $\beta$ ) were measured in the supernatants with enzyme-linked immunosorbent assay. Migration of MSC and HUVEC induced by the supernatants was evaluated in Boyden chambers.

Results More TGF- 31 was released (mean $\pm \mathrm{SD}$ in $\mathrm{pg} / \mathrm{mL}$ of blood) from L-PRF $(37,796 \pm 5492)$ compared with L-PRP $(23,738 \pm 6848 ; \quad \mathrm{p}<0.001)$ and blood clot (3739 $\pm 4690 ; p<0.001)$, whereas more VEGF and IL-1ß were released from blood clot (1933 \pm 704 and $2053 \pm 908$, respectively) compared with both L-PRP (642 \pm 208 ; $\mathrm{p}<0.001$ and $273 \pm 386 ; \mathrm{p}<0.001$, respectively) and L-PRF $(852 \pm 376$; $p<0.001$ and $65 \pm 56, \mathrm{p}<0.001$, respectively). No differences were observed in IGF-1 and PDGF-AB released from any of the concentrates. TGF- $\beta 1$ release peaked at Day 7 in L-PRF and at 8 hours and Day 7 in L-PRP and 8 hours and Day 14 in blood clot. In all concentrates, main release of VEGF occurred between 3 and 7 days and of IL- $1 \beta$ between Days 1 and 7. IGF-1 and PDGF-AB were released until Day 1 in L-PRP and blood clot, in contrast to sustained release over the first 3 days in L-PRF. The strongest migration of MSC occurred in response to L-PRF, and more HUVEC migration was seen in L-PRF and blood clot compared with L-PRP. TGF- $\beta 1$ correlated with initial platelet counts in L-PRF (Pearson $r=0.66, p=0.0273$ ) and initial leukocyte counts in L-PRP (Pearson $r=0.83$, $p=0.0016)$. A positive correlation of IL- $1 \beta$ on migration of 
MSC and HUVEC was revealed (Pearson $r=0.16$, $\mathrm{p}=0.0208$; Pearson $\mathrm{r}=0.31, \mathrm{p}<0.001)$.

Conclusions In comparison to L-PRP, L-PRF had higher amounts of released TGF- $\beta 1$, a long-term release of growth factors, and stronger induction of cell migration. Future preclinical studies should confirm these data in a defined injury model.

Clinical Relevance By characterizing the biologic properties of different platelet concentrates in vitro, we may gain a better understanding of their clinical effects and develop guidelines for specific future applications.

\section{Introduction}

The use of platelet concentrates as a therapeutic treatment to enhance healing and control inflammation has gained attention in sports medicine and orthopaedics [10]. By facilitating recruitment, proliferation, and maturation of cells participating in regeneration of tendon, ligaments, bone, and cartilage, platelet concentrates may prove beneficial for tissues with restricted blood supply, slow cell turnover, and limited extracellular matrix restoration [4]. In early stages of wound healing after surgical interventions or bone fractures, the coagulation cascade activates platelets. Subsequently, the content of their granules is released into the wound site, simultaneously delivering a plethora of autologous growth factors in physiologic proportions to the affected site and contributing to the healing process.

The advantages of platelet concentrates are their autologous nature, simple collection, easy bedside preparation, and clinical application without the risks associated with allogenic products. Currently, various preparations of platelet concentrates are being applied in sports medicine [8, 10, 15, 19, 21, 27-29], dental and maxillofacial surgery [9], in wound healing [16], and veterinary medicine [4, 30]. Based on the presence of leukocytes and fibrin architecture, platelet concentrates are classified in four main families: pure platelet-rich plasma (P-PRP) without leukocytes and with a low-density fibrin network after activation; leukocyte- and platelet-rich plasma (L-PRP), with leukocytes and with a low-density fibrin network after activation; pure platelet-rich fibrin $(\mathrm{P}-$ PRF) without leukocytes and with a high-density fibrin network; and leukocyte- and platelet-rich fibrin (L-PRF) with leukocytes and a high-density fibrin network [10]. Platelet concentrate preparations differ substantially in the amount and dynamics of growth factor release [4, 6, 14, 23]. During the initial phase of wound healing, platelets interact with the fibrin clot to form a hemostatic plug and to create a temporary scaffold to capture cytokines and support and stimulate cell migration and proliferation [1]. This dense, platelet-rich fibrin construct may allow for sustained release of growth factors as demonstrated in previous studies with L-PRF [13, 37] and with platelet-rich fibrin matrix and platelet-rich fibrin membrane compared with blood clot [33]. The plethora of different concentrates together with various preparation protocols represents the main hurdle to understand the often unclear clinical results. By characterizing the biologic properties of L-PRF and L-PRP, the most often used platelet-rich concentrates [7], in comparison to the naturally forming blood clot in an in vitro study, we may begin to better understand how to characterize their clinical effects.

The aims of this study were to (1) analyze the concentration and kinetics of growth factors-transforming growth factor $\beta 1$ (TGF- $\beta 1$ ), vascular endothelial growth factor (VEGF), insulin-like growth factor-1 (IGF-1), plateletderived growth factor $\mathrm{AB}$ (PDGF-AB) and interleukin-1B (IL-1ß)-released from L-PRF, L-PRP, and blood clot; (2) investigate the migration of mesenchymal stem cells (MSC) and endothelial cells as a functional response to the factors released; and (3) uncover correlations between individual growth factors with the initial platelet/leukocyte counts or the induced cell migration.

\section{Materials and Methods}

Our study was conducted in agreement with the guidelines of the institutional ethical committee (Kantonale Ethikkommision, Bern, Switzerland). Peripheral venous blood (65 mL) was collected from 11 healthy men volunteers (age range, 2738 years; mean, 32.6 years) on signed informed consent. Platelets and leukocytes were counted with cell counter Sysmex KX-21 N (Sysmex-Suisse AG, Horgen, Switzerland). The average platelet and leukocyte counts per microliter of blood were $222 \pm 71 \times 103$ and $5954 \pm 723$, respectively. L-PRF, L-PRP, and blood clot were prepared from each donor concurrently (a single-donor model) to ensure minimal introduction of potential variables.

To prepare L-PRF, $9 \mathrm{~mL}$ of blood was collected in 10$\mathrm{mL}$ glass-coated, plastic tubes (Vacutainer; BD Biosciences, Allschwil, Switzerland) and immediately centrifuged at $400 \times \mathrm{g}$ for 12 minutes at room temperature using a table centrifuge specifically designed for this application (EBA 20; Andreas Hettich $\mathrm{GmbH} \& \mathrm{Co} \mathrm{KG}$, Tuttlingen, Germany) [38] (Fig. 1A). L-PRP was prepared according to the manufacturer's protocol (Biomet Inc, Dietikon, Switzerland) (Fig. 1C). Briefly, $27 \mathrm{~mL}$ of blood was collected in a $30-\mathrm{mL}$ syringe containing $3 \mathrm{~mL}$ of adenosine-citrate-dextrose acid (ACD-A) anticoagulant. The content of the syringe was transferred into the $30-\mathrm{mL}$ GPS III separation tube (Biomet Inc) and centrifuged for 15 minutes at $1900 \times \mathrm{g}$ at room temperature. After 
Fig. 1A-C Schematic overview of the protocols to prepare platelet concentrates is shown. Each collected blood sample was divided and processed according to the depicted protocol to obtain (A) L-PRF; (B) blood clot, and (C) L-PRP concentrates.
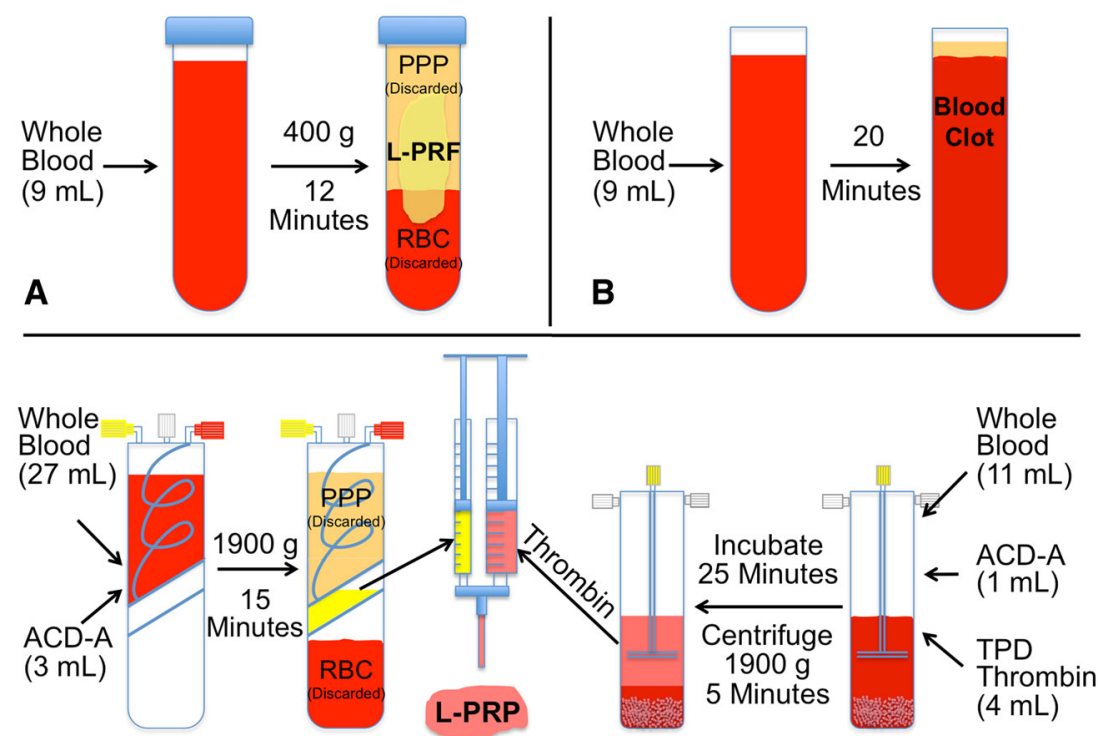

C

removing the plasma, the buffy coat was resuspended in the leftover plasma by shaking the tube for 30 seconds. A second syringe containing $1 \mathrm{~mL}$ of ACD-A was used to collect an additional $11 \mathrm{~mL}$ of blood, which was transferred into a Clotalyst ${ }^{\circledR}$ disposable tube (Biomet Inc) containing $4 \mathrm{~mL}$ of $\mathrm{TPD}^{\mathrm{TM}}$ Thrombin Reagent (Biomet Inc). After gentle mixing, the tube was placed into a Clotalyst ${ }^{\mathbb{R}}$ heater for 25 minutes. Subsequently, the mix was centrifuged for 5 minutes at $1900 \times \mathrm{g}$. Coagulation was performed using a double syringe (spray applicator), allowing uniform mixing of the two components, resulting in formation of a clot in a six-well cell culture plate (BD Biosciences, Allschwil, Switzerland) . To prepare blood clot, $9 \mathrm{~mL}$ of blood was collected in 10-mL glass-coated, plastic tubes (Fig. 1B). The closing lid was removed and the blood was allowed to coagulate for 20 minutes at room temperature. L-PRF, L-PRP, and blood clot were placed in separate wells of six-well plates, covered with $6 \mathrm{~mL}$ of culture medium (Iscove's Modified Dulbecco's Medium [IMDM], L-glutamine, and $1 \%$ penicillin/streptomycin), without addition of fetal bovine serum (FBS), and incubated at $37^{\circ} \mathrm{C}$ in a humidified atmosphere with $5 \% \mathrm{CO}_{2}$ for 28 days. At preparation, the platelet concentrates showed macroscopic differences, with L-PRF being more consistent, fibrous, and of less reddish appearance as a result of removal of red blood cells compared with the more gelatinous appearance of L-PRP and blood clot (Fig. 2). L-PRF and blood clot retained their initial appearance after 28 days in culture; however, L-PRP partially disintegrated. During culture, entire medium was collected after 8 hours and $1,3,7,14$, and 28 days, and an equal volume of fresh medium was added back to each well. All collected culture supernatants were stored at $-80^{\circ} \mathrm{C}$ before analysis. Culture

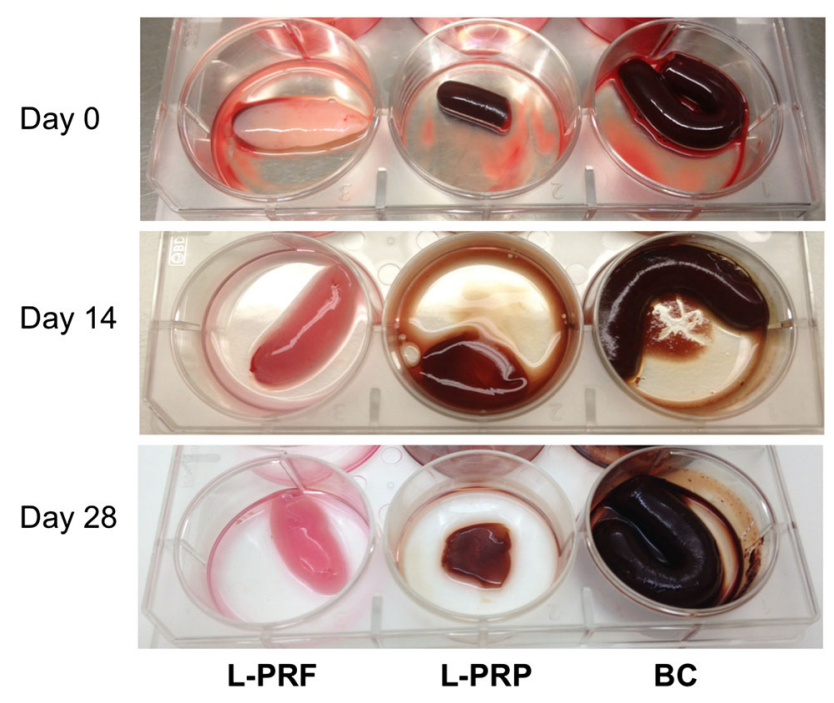

Fig. 2 The appearances of L-PRF, L-PRP, and blood clot (BC) during culture are shown. L-PRF, L-PRP, and blood clot concentrates were cultured in six-well plates for 28 days. The appearance of each concentrate at the time of preparation (Day 0), after Day 14, and Day 28 is shown.

supernatants were analyzed for concentration of TGF- $\beta 1$, VEGF, IGF-1, PDGF-AB, and IL-1ß and for their capacity to induce migration of human bone marrow MSC and primary human umbilical vein endothelial cells (HUVEC).

The levels of TGF-B1, VEGF, IGF-1, PDGF-AB, and IL$1 \beta$ were quantified in culture supernatants using commercially available enzyme-linked immunosorbent assay (ELISA duo sets; R\&D Systems, Abingdon, UK) kits according to the manufacturer's instructions. To detect the total amount of TGF- $\beta 1$, the latent form of TGF- $\beta 1$ was first converted into the active form according to the manufacturer's instructions. The 
absorbance was measured with a Tecan Infinite 200 Pro plate reader (Tecan Group Ltd, Männedorf, Switzerland) at $450 \mathrm{~nm}$ with a reference wavelength of $540 \mathrm{~nm}$. Standard curves were generated using standards supplied with each kit. Samples were tested in triplicate, and the absorbance from wells containing medium only were set as the blank and subtracted from the test well readout.

Cell migration was performed with human bone marrow-derived MSC and HUVEC from our cell bank [17, 18], cultured in DMEM/F-12 (Life Technologies, Basel, Switzerland), 10\% FBS (Hyclone, Lausanne, Switzerland), $100 \mathrm{U} / \mathrm{mL}$ penicillin $+100 \mathrm{mg} / \mathrm{mL}$ streptomycin (Life Technologies) in a $5 \% \mathrm{CO}_{2}$, and $95 \%$ air-humidified incubator at $37^{\circ} \mathrm{C}$. Culture medium was replaced with medium without FBS 24 hours before the migration assay. On trypsinization and washing, cells were labeled with Calcein AM (R\&D Systems, Abingdon, UK) for 30 minutes, washed, and resuspended in medium. The migration assay was performed in Boyden chambers (Neuroprobe, Gaithersburg, MD, USA) with medium supernatant samples collected at different time points. The polycarbonated filter ( $8-\mu \mathrm{m}$ pore size, $5.6 \mathrm{~mm}$ diameter; Neuroprobe) of the Boyden chamber was precoated with fibronectin (10 $\mu \mathrm{g} / \mathrm{mL}$; Sigma, Buchs, Switzerland) for 60 minutes at $37^{\circ} \mathrm{C}$, then washed twice with deionized water, and dried. Twenty-nine microliters of each sample were placed into the lower chamber as a chemoattractant. IMDM containing $10 \%$ FBS was used as a reference. The polycarbonated precoated filter was then placed on top of the lower chamber and seeded with 20,000 Calcein AM stained cells in $50 \mu \mathrm{L}$. To allow cell migration, chambers were incubated for 4 hours in the incubator. After removing the nonmigrated cells from the top of the filter with a cotton swab, fluorescence emission was measured at $580 \mathrm{~nm}$ with excitation at $485 \mathrm{~nm}$ in a bottom-reading fluorescent Tecan Infinite 200 Pro plate reader (Tecan Group Ltd). The migration index was defined as the ratio of number of cells migrating toward the tested samples containing the growth factors released by L-PRF, L-PRP, or blood clot divided by the number of cells migrating toward media containing $10 \%$ FBS. Each assay was performed in triplicate, and two migration experiments were performed for each cell type.

Statistical analysis was performed using Prism Version 6 for Macintosh (GraphPad Software, Inc, San Diego, CA, USA). All data were normalized to the amount of supernatant collected from L-PRF, L-PRP, and blood clot at each time point. Data are expressed as mean $\pm \mathrm{SD}$. The significance of differences among means of data of total growth factor release values were analyzed using one-way analysis of variance (ANOVA) and Bonferroni post hoc and the growth factor release kinetics and migration measurements by two-way repeated measures ANOVA and Bonferroni post hoc test. Pearson correlation was applied to analyze potential correlations between: (1) initial platelet or leukocyte counts in blood and the total concentration of the different growth factor released; and (2) growth factor concentration and cell migration. A $\mathrm{p}$ value $<0.05$ was considered statistically significant.

\section{Results}

Differential growth factor concentration and dynamics of release were observed among the three platelets preparations. Analysis of total growth factor release (Fig. 3) revealed that more TGF- $\beta 1$ was released (in $\mathrm{pg} / \mathrm{mL}$ of blood) from L-PRF $(37,796 \pm 5492)$ compared with L-PRP $(23,738 \pm 6848 ; \mathrm{p}<0.001)$ or blood clot $(23,739 \pm 4690$; $\mathrm{p}<0.001)$, whereas more VEGF and IL-1ß were released from blood clot $(1933 \pm 704$ and $2053 \pm 908$, respectively) compared with both L-PRP $(642 \pm 208 ; \mathrm{p}<0.001$ and $273 \pm 386 ; \mathrm{p}<0.001$, respectively) and L-PRF (852 \pm $376 ; \mathrm{p}<0.001$ and $65 \pm 56 ; \mathrm{p}<0.001$, respectively). In contrast, no differences were noted for the amounts of IGF-1 and PDGF-AB released from any platelet concentrate. Analysis of the kinetics of the growth factors released showed that TGF- 31 had bimodal release in L-PRP and blood clot with peaks at 8 hours and 7 days for L-PRP and 8 hours and 14 days for blood clot (Fig. 4). In contrast, a single peak at 7 days was observed for L-PRF. VEGF release from L-PRP peaked at 8 hours and 3 days in contrast with L-PRF and blood clot, where VEGF peaked at 7 days. IGF-1 was almost completely released within the first 8 hours from L-PRP and gradually released during the first 3 days from L-PRF and blood clot. Similarly, almost all PDGF-AB was released during the first 8 hours from L-PRP and blood clot in contrast to a gradual release from L-PRF. Release of IL- $1 \beta$ peaked at 1 day in L-PRF and blood clot and at 3 days in L-PRP.

MSC and HUVEC migration in response to L-PRP, L-PRF, and blood clot revealed differential migration to individual platelet concentrates at the different time points analyzed. MSC migrated mainly at Day 1 for L-PRP and blood clot and at Day 3 for L-PRF (Fig. 5). Higher migration was measured (fold change over FBS migration) for L-PRF compared with L-PRP at Day 3 (1.29 \pm 0.27 versus $0.84 \pm 0.44, \quad \mathrm{p}=0.0025)$, Day $7(1.05 \pm 0.27$ versus $0.51 \pm 0.44, \mathrm{p}<0.001)$, and at Day $14(0.74 \pm 0.23$ versus $0.36 \pm 0.35, \mathrm{p}=0.0226$ ) and compared with blood clot at Day $7(1.05 \pm 0.27$ versus $0.67 \pm 0.17, \mathrm{p}=$ 0.0195). Migration of HUVEC peaked at Day 1 for L-PRP and between Day 1 and Day 3 for L-PRF and blood clot. Higher migration was detected for L-PRF at 8 hours compared with L-PRP $(0.82 \pm 0.28$ versus $0.18 \pm 0.12$, $\mathrm{p}<0.001)$ and blood clot $(0.82 \pm 0.28$ versus $0.43 \pm$ $0.16, \mathrm{p}=0.0001$ ), and remained higher for L-PRF and blood clot compared with L-PRP at Day $3(1.16 \pm 0.19$ 


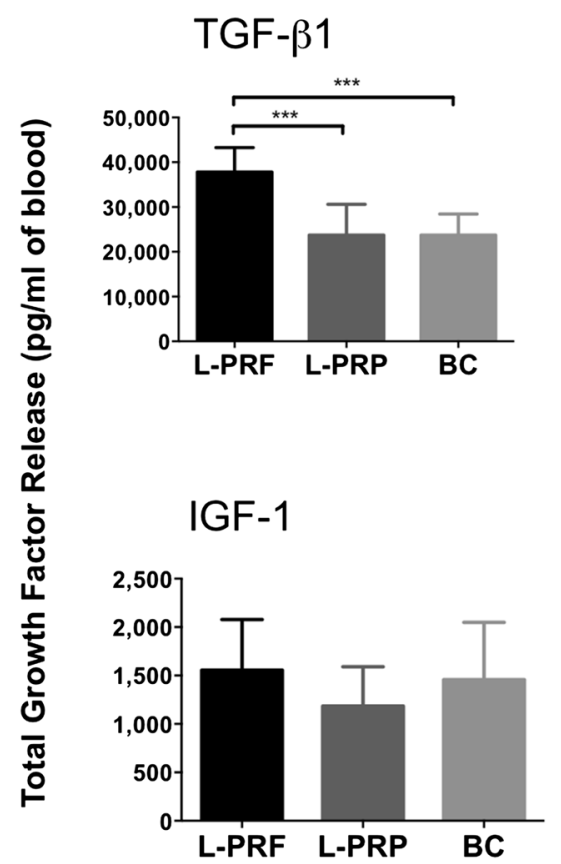

Fig. 3 Total growth factor release from L-PRF, L-PRP, and blood clot (BC) is shown. The total amount of TGF- 1 1, VEGF, IGF-1, PDGF-AB, and $I L-1 \beta$ released during the entire culturing period was determined by summing up the release of each growth factor measured in media cultured with L-PRF, L-PRP, or blood clot at each time point ( 8 hours

versus $0.59 \pm 0.25, \mathrm{p}<0.001$ and $1.09 \pm 0.16$ versus $0.59 \pm 0.25, \mathrm{p}<0.001$, respectively), Day $7(0.76 \pm 0.17$ versus $0.24 \pm 0.22, \mathrm{p}<0.001$ and $0.70 \pm 0.33$ versus $0.24 \pm 0.22, \mathrm{p}<0.001$, respectively) and Day $14(0.42 \pm$ 0.14 versus $0.14 \pm 0.14, \mathrm{p}=0.0169$ and $0.62 \pm 0.19$ versus $0.14 \pm 0.14, \mathrm{p}<0.001$, respectively).

Pearson's correlation coefficient analysis of platelet or leukocyte counts in the collected blood before platelet preparation and the total amount of the individual growth factors released by the three platelet-rich concentrates revealed a correlation for TGF- $\beta 1$ and platelet counts from L-PRF (Pearson $r=0.66, p=0.0273$ ) and leukocyte counts and TGF- $\beta 1$ from L-PRP (Pearson $r=0.83, p=$ 0.0016). Pearson's correlation coefficient analysis of the concentration of individual growth factors at different time points during the 28 days of in vitro culture obtained for all platelet concentrates and the MSC or HUVEC migration revealed a positive correlation of IL-1 $\beta$ on migration of both cell types and a negative correlation of PDGF-AB on both cell types, IGF-1 on MSC, and TGF- $\beta 1$ on HUVEC (Table 1). Release of VEGF did not influence the migration of either cell type.

\section{Discussion}

Platelet-rich concentrates have been increasingly used to enhance tissue healing by concentrating growth factors at and $1,3,7,14$, and 28 days). Data are presented as mean \pm SD from triplicate measurements of 11 samples as total amount normalized to the volume of blood used for the preparation of the platelet concentrate. Statistical evaluation was done using one-way ANOVA and Bonferroni post hoc test. Significant differences are indicated: ***p $<0.001$.

the injury site. These concentrates contain a mixture of anabolic factors (derived from platelets) and catabolic factors (produced by leukocytes), yet their concentrations vary across many different preparation systems. Studies investigating growth factor concentration and release kinetics as a consequence of different platelet concentrate preparations have been previously reported $[6,13,20,24,25,32,33,35$, 37]. However, release of growth factors from L-PRF and L-PRP compared with natural blood clot-a physiologic source of growth factors in any wound-and their influence on cell migration has not been investigated. Our data demonstrate more TGF- $\beta 1$ release from L-PRF and more VEGF and IL-1 $\beta$ release from blood clot. The dynamics of release demonstrate sustained release of all factors from L-PRF. Migration of MSC and HUVEC in response to L-PRP, L-PRF, and blood clot reveals no difference in the overall patterns over time but differential responses to each platelet concentrate at different time points. The strongest migration of MSC occurred in response to L-PRF, and more HUVEC migration was seen in L-PRF and blood clot compared with L-PRP. Finally, we uncovered a correlation between platelet counts in blood and TGF- $\beta 1$ released from L-PRF and leukocyte counts and TGF- $\beta 1$ released from L-PRP as well as a positive correlation of IL-1 $\beta$ on migration of MSC and HUVEC.

The main limitation of our study is the artificial in vitro system used for culturing the concentrates. In vivo, a 


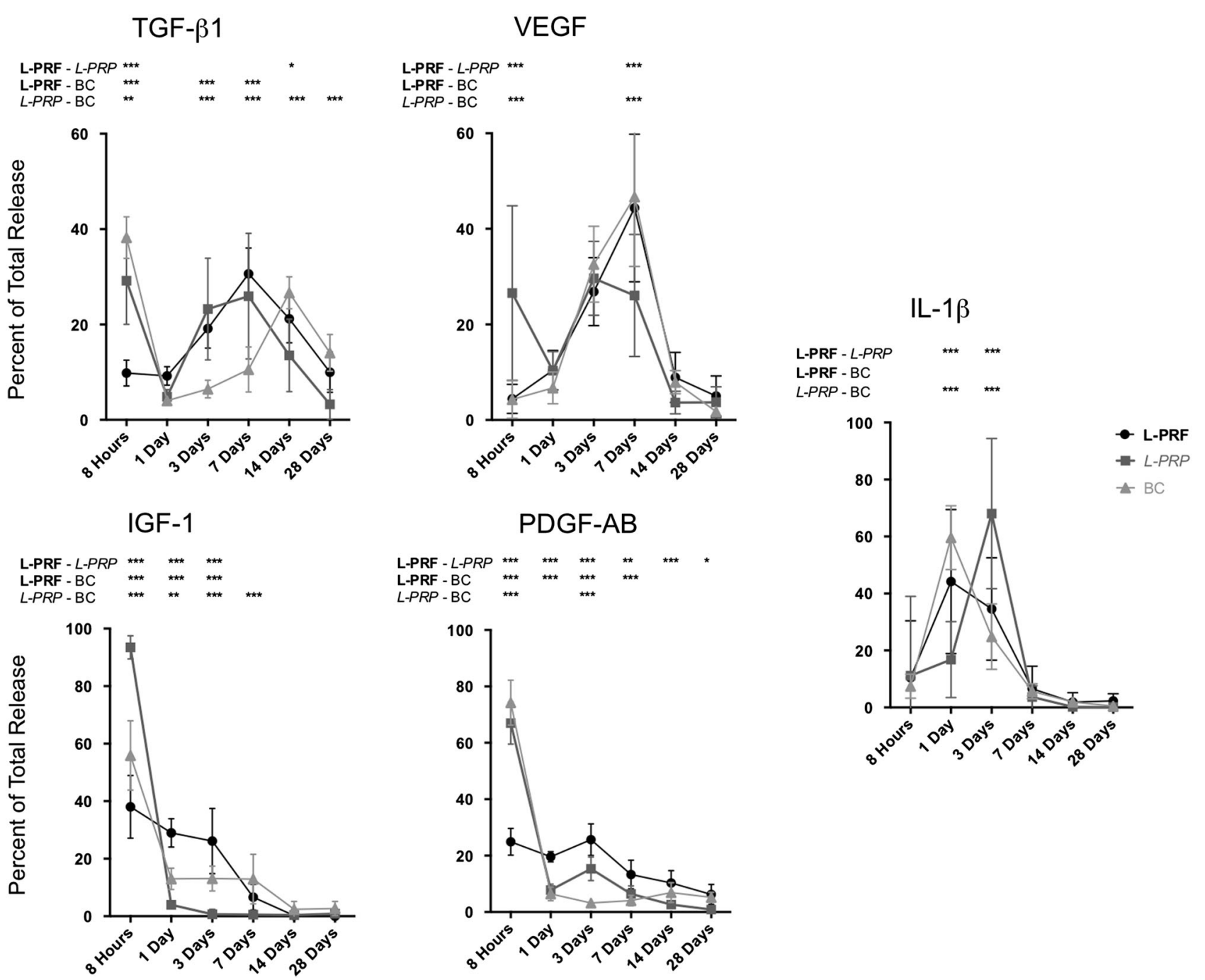

Fig. 4 Kinetics of growth factor release from L-PRP, L-PRF, and blood clot (BC) are shown. Release of TGF-ß1, VEGF, IGF-1, PDGF$\mathrm{AB}$, and IL-1 1 from cultured L-PRF, L-PRP, or BC was determined at each time point ( 8 hours, $1,3,7,14$ and 28 days). The amounts released are expressed as a percentage of total release per time point.

physiologic environment of the tissue would influence the behavior of platelet concentrate in terms of structure, cellular crosstalk, exposure to degradation enzymes, and release of growth factors. However, characterization of platelets in vitro remains an important step toward the understanding of their effects in vivo. For example, the knowledge that L-PRF releases high amounts of TGF- $\beta 1$ and allows sustained release of other growth factors could provide important guidelines for the choice of tissue and/or injury in future preclinical and ultimately clinical studies. Another limitation is the characterization of only two leukocyte-rich platelet concentrates, L-PRP and L-PRF. However, these two families of platelet concentrates are the most often used technologies in the field of regenerative medicine [7].
Data are presented as mean \pm SD from triplicate measurements of 11 samples. Statistical evaluation was done using two-way repeated ANOVA and Bonferroni post hoc test. Significant differences among the platelet concentrates for each factor at different time points are indicated: $* \mathrm{p}<0.05, * * \mathrm{p}<0.01, * * * \mathrm{p}<0.001$.

Release patterns of the growth factors analyzed in our study show some important differences. The highest TGF$\beta 1$ release occurred from L-PRF and the highest IL-1 $\beta$ and VEGF release from blood clot, whereas no differences were noted for PDGF-AB and IGF-1 release across the concentrates. L-PRP released most of the growth factors at the beginning of culture in contrast to more sustained release from L-PRF. These release dynamics have been previously observed for TGF- $\beta 1$, VEGF, IGF- 1 , and PDGF-AB in L-PRF [37]. In comparison to another study [20], the release patterns of TGF- $\beta 1$ in L-PRP and L-PRF proved similar, yet those of PDGF-AB differed. Several factors can influence the total release as well as the dynamics of growth factor release from platelet concentrates. Structure of the fibrin network and concentration of leukocytes are known to 
HUVEC

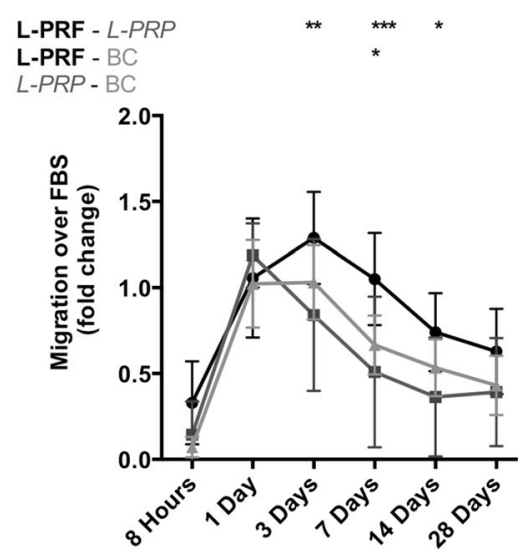

Fig. 5 Migration of MSC and HUVEC in response to factors released from L-PRF, L-PRP, and blood clot (BC) is shown. Migration of MSC and HUVEC was assessed in Boyden chambers with media collected after 8 hours and 1, 3, 7, 14 and 28 days of cultured L-PRP, L-PRF, and blood clot compared with media containing $10 \%$ FBS and expressed as fold change.

influence growth factor release from platelet concentrates [12]. Fast clotting of L-PRP resulting from the addition of thrombin is in sharp contrast to the slow clotting in L-PRF and blood clot. In our study, L-PRP formed an unstable matrix that disintegrated during culture and led to an initial burst release of growth factors, as previously shown [13, 20, 32]. In contrast, the more structured fiber network-formed during progressive L-PRF polymerization-led to a more sustained growth factor release, in line with previous studies $[12,13,20,24,33,37]$. Whereas the immediate release of IGF-1 and PDGF-AB could be attributed to simple diffusion from plasma (IGF-1) or instant release from activated platelets (IGF- 1 and PDGF-AB), the late release of TGF- $\beta 1$ and VEGF could be explained by the production of growth factors by the leukocytes present in the three platelet concentrates [12].

Cell migration plays a crucial role in the healing process. MSC represent a cell pool that rebuilds damaged tissue and endothelial cells contribute to angiogenesis. Migration patterns induced by culture supernatant of platelets concentrates in our study do not differ between the two cell types, although overall L-PRP induced less migration at later time points. The strongest migration of MSC was seen in response to L-PRF, and more HUVEC migration was seen in L-PRF and blood clot compared with L-PRP.

L-PRP and L-PRF are described as concentrates rich in leukocytes [10]. The beneficial or detrimental effect of leukocyte remains controversial $[2,31]$. Leukocyte production of catabolic cytokines in L-PRP raises concerns [5, $31]$, whereas their antimicrobial effects $[3,11]$ and release of growth factors and matrix proteins from L-PRF for more than 7 days indicate their beneficial effect $[2,12,13,20]$.

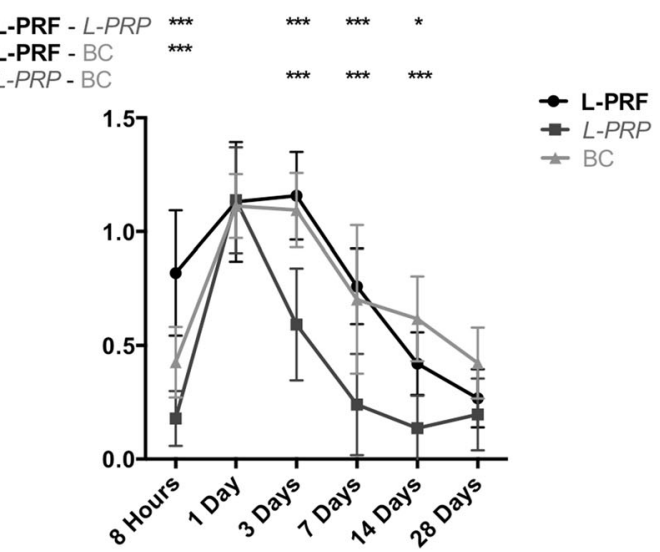

Data are presented as mean \pm SD from triplicate measurements of 11 samples. Statistical evaluation was done using two-way repeated ANOVA and Bonferroni post hoc test. Significant differences for MSC and HUVEC migration among the platelet concentrates at different time points are indicated: $* \mathrm{p}<0.05, * * \mathrm{p}<0.01, * * \mathrm{p} p<0.001$.

Although L-PRP and L-PRF are rich in leukocytes, we observed higher total amounts of IL-1 $\beta$ and VEGF in blood clot compared with L-PRF and L-PRP, suggesting a partial loss of leukocytes during L-PRP and L-PRF preparations. IL-1 $\beta$ has been previously correlated with the number of neutrophils and monocytes in L-PRP concentrate [31]. Similarly, VEGF has been correlated with the number of leukocytes [66], although VEGF is also released from platelets [22]. Previous work has shown a correlation between platelet concentration in PRP and TGF- $\beta 1$ release [31]. In our study, more TGF-ß1 was released from L-PRF compared with L-PRP and blood clot, which may suggest that L-PRF provides higher platelet enrichment. Although we could not compare platelet counts across individual concentrates as a result of the solid character of L-PRF and blood clot, TGF- 31 was the only factor for which, in L-PRF, we found a correlation with platelet counts in the collected blood. These data suggest that preparation of L-PRF represents a more reproducible system to concentrate platelets. On the other hand, the amount of TGF- $\beta 1$ released by L-PRP correlated with the blood leukocyte count, emphasizing the role of leukocytes as a source of growth factors in platelet concentrates. The correlation between the presence of growth factors in the concentrates and the induced cell migration patterns revealed contradictory results. As previously described using the single recombinant protein, TGF- $\beta 1$ had no effect on MSC migration [36] and negatively correlated with HUVEC migration [34]. However, the negative correlation uncovered between PDGF-AB and cell migration appears to be at odds with an earlier study [26], in which recombinant human PDGF-AB induced migration of MSC. The discrepancy in the results could be due to a 
Table 1. Correlation between growth factor release and cell migration

\begin{tabular}{|c|c|c|c|c|c|c|c|c|c|c|}
\hline \multirow[t]{3}{*}{ Cell migration } & \multicolumn{10}{|c|}{ Growth factor concentration } \\
\hline & \multicolumn{2}{|c|}{ TGF- $\beta 1$} & \multicolumn{2}{|c|}{ VEGF } & \multicolumn{2}{|l|}{ IGF-1 } & \multicolumn{2}{|c|}{ PDGF-AB } & \multicolumn{2}{|c|}{ IL-1 $\beta$} \\
\hline & $\mathrm{r}$ & $\mathrm{p}$ value & $\mathrm{r}$ & $\mathrm{p}$ value & $\mathrm{r}$ & $\mathrm{p}$ value & $\mathrm{r}$ & $\mathrm{p}$ value & $\mathrm{r}$ & $\mathrm{p}$ value \\
\hline MSC & -0.12 & 0.09 & 0.06 & 0.39 & -0.20 & 0.005 & -0.53 & $<\mathbf{0 . 0 0 1}$ & 0.16 & 0.02 \\
\hline HUVEC & -0.34 & $<0.001$ & 0.14 & 0.05 & -0.07 & 0.31 & -0.21 & $\mathbf{0 . 0 0 3}$ & 0.31 & $<0.001$ \\
\hline
\end{tabular}

Growth factor concentrations obtained from L-PRF, L-PRP, and blood clot supernatants at 8 hours and 1, 3, 7, 14, and 28 days by enzyme-linked immunosorbent assay were evaluated for correlation with migration values obtained from MSC or HUVEC by Boyden chamber assay with the same supernatants. A total of 198 values was used for the evaluation per cell type and growth factor. Pearson correlation coefficients (r) and $p$ values are shown. Significant correlations are in bold; TGF- $\beta 1=$ tumor growth factor $1 \beta$; VEGF = vascular endothelial growth factor; IGF$1=$ insulin-like growth factor; PDGF-AB = platelet-derived growth factor AB; IL- $1 \beta=$ interleukin $1 \beta$; MSC = mesenchymal stem cells; HUVEC $=$ human umbilical vein endothelial cells

comparison of the effect of a pure recombinant protein versus a cocktail of factors present in platelet concentrates. Furthermore, IL-1 $\beta$, which is not secreted by platelets, was the only factor that appeared to induce cell migration. These results underline the complexity of growth factor and cell interactions in platelet concentrates and the importance of understanding the mechanisms governing the cellular process during wound healing.

In summary, L-PRF may offer advantages over L-PRP comprising overall higher amounts of released TGF- $\beta 1$, a sustained, long-term release of investigated growth factors, and stronger induction of cell migration in vitro. By determining in vitro the content, amount, and release kinetics of growth factors in different platelet-rich concentrates, we may start to understand controversial clinical results and develop guidelines for future specific applications, where a particular concentrate could prove more appropriate for a certain type of tissue and/or injury.

Acknowledgments We thank Biomet Inc for providing GPS III kits.

\section{References}

1. Ahmed TA, Dare EV, Hincke M. Fibrin: a versatile scaffold for tissue engineering applications. Tissue Eng Part B Rev. 2008;14:199-215.

2. Bielecki T, Dohan Ehrenfest DM, Everts PA, Wiczkowski A. The role of leukocytes from L-PRP/L-PRF in wound healing and immune defense: new perspectives. Curr Pharm Biotechnol. 2012;13:1153-1162.

3. Bielecki TM, Gazdzik TS, Arendt J, Szczepanski T, Krol W, Wielkoszynski T. Antibacterial effect of autologous platelet gel enriched with growth factors and other active substances: an in vitro study. J Bone Joint Surg Br. 2007;89:417-420.

4. Boswell SG, Cole BJ, Sundman EA, Karas V, Fortier LA. Platelet-rich plasma: a milieu of bioactive factors. Arthroscopy. 2012;28:429-439.

5. Boswell SG, Schnabel LV, Mohammed HO, Sundman EA, Minas $\mathrm{T}$, Fortier LA. Increasing platelet concentrations in leukocytereduced platelet-rich plasma decrease collagen gene synthesis in tendons. Am J Sports Med. 2014;42:42-49.
6. Castillo TN, Pouliot MA, Kim HJ, Dragoo JL. Comparison of growth factor and platelet concentration from commercial platelet-rich plasma separation systems. Am J Sports Med. 2011;39:266-271.

7. Cieslik-Bielecka A, Dohan Ehrenfest DM, Lubkowska A, Bielecki T. Microbicidal properties of leukocyte- and platelet-rich plasma/fibrin (L-PRP/L-PRF): new perspectives. J Biol Regul Homeost Agents. 2012;26:43S-52S.

8. Dallari D, Savarino L, Stagni C, Cenni E, Cenacchi A, Fornasari PM, Albisinni U, Rimondi E, Baldini N, Giunti A. Enhanced tibial osteotomy healing with use of bone grafts supplemented with platelet gel or platelet gel and bone marrow stromal cells. $J$ Bone Joint Surg Am. 2007;89:2413-2420.

9. Del Fabbro M, Bortolin M, Taschieri S, Weinstein R. Is platelet concentrate advantageous for the surgical treatment of periodontal diseases? A systematic review and meta-analysis. $J$ Periodontol. 2011;82:1100-1111.

10. Dohan Ehrenfest DM, Andia I, Zumstein MA, Zhang CQ, Pinto NR, Bielecki T. Classification of platelet concentrates (plateletrich plasma-PRP, platelet-rich fibrin-PRF) for topical and infiltrative use in orthopedic and sports medicine: current consensus, clinical implications and perspectives. Muscles Ligaments Tendons J. 2014;4:3-9.

11. Dohan Ehrenfest DM, Bielecki T, Del Corso M, Inchingolo F, Sammartino G. Shedding light in the controversial terminology for platelet-rich products: platelet-rich plasma (PRP), plateletrich fibrin (PRF), platelet-leukocyte gel (PLG), preparation rich in growth factors (PRGF), classification and commercialism. $J$ Biomed Mater Res A. 2010;95:1280-1282.

12. Dohan Ehrenfest DM, Bielecki T, Jimbo R, Barbe G, Del Corso M, Inchingolo F, Sammartino G. Do the fibrin architecture and leukocyte content influence the growth factor release of platelet concentrates? An evidence-based answer comparing a pure platelet-rich plasma (P-PRP) gel and a leukocyte- and platelet-rich fibrin (L-PRF). Curr Pharm Biotechnol. 2012;13:1145-1152.

13. Dohan Ehrenfest DM, de Peppo GM, Doglioli P, Sammartino G. Slow release of growth factors and thrombospondin-1 in Choukroun's platelet-rich fibrin (PRF): a gold standard to achieve for all surgical platelet concentrates technologies. Growth Factors. 2009;27:63-69.

14. Dragoo JL, Braun HJ, Durham JL, Ridley BA, Odegaard JI, Luong R, Arnoczky SP. Comparison of the acute inflammatory response of two commercial platelet-rich plasma systems in healthy rabbit tendons. Am J Sports Med. 2012;40:1274-1281.

15. Foster TE, Puskas BL, Mandelbaum BR, Gerhardt MB, Rodeo SA. Platelet-rich plasma: from basic science to clinical applications. Am J Sports Med. 2009;37:2259-2272. 
16. Frykberg RG, Driver VR, Carman D, Lucero B, Borris-Hale C, Fylling CP, Rappl LM, Clausen PA. Chronic wounds treated with a physiologically relevant concentration of platelet-rich plasma gel: a prospective case series. Ostomy Wound Manage. 2010;56:36-44.

17. Giovannini S, Brehm W, Mainil-Varlet P, Nesic D. Multilineage differentiation potential of equine blood-derived fibroblast-like cells. Differentiation. 2008;76:118-129.

18. Giovannini S, Diaz-Romero J, Aigner T, Heini P, Mainil-Varlet $\mathrm{P}$, Nesic D. Micromass co-culture of human articular chondrocytes and human bone marrow mesenchymal stem cells to investigate stable neocartilage tissue formation in vitro. Eur Cell Mater. 2010;20:245-259.

19. Hall MP, Band PA, Meislin RJ, Jazrawi LM, Cardone DA. Platelet-rich plasma: current concepts and application in sports medicine. J Am Acad Orthop Surg. 2009;17:602-608.

20. He L, Lin Y, Hu X, Zhang Y, Wu H. A comparative study of platelet-rich fibrin (PRF) and platelet-rich plasma (PRP) on the effect of proliferation and differentiation of rat osteoblasts in vitro. Oral Surg Oral Med Oral Pathol Oral Radiol Endod. 2009;108:707-713.

21. Lopez-Vidriero E, Goulding KA, Simon DA, Sanchez M, Johnson DH. The use of platelet-rich plasma in arthroscopy and sports medicine: optimizing the healing environment. Arthroscopy. 2010;26:269-278.

22. Maloney JP, Silliman CC, Ambruso DR, Wang J, Tuder RM, Voelkel NF. In vitro release of vascular endothelial growth factor during platelet aggregation. Am J Physiol. 1998;275:H1054-1061.

23. Mazzocca AD, McCarthy MB, Chowaniec DM, Cote MP, Romeo AA, Bradley JP, Arciero RA, Beitzel K. Platelet-rich plasma differs according to preparation method and human variability. $J$ Bone Joint Surg Am. 2012;94:308-316.

24. Mazzucco L, Balbo V, Cattana E, Guaschino R, Borzini P. Not every PRP-gel is born equal. Evaluation of growth factor availability for tissues through four PRP-gel preparations: Fibrinet, RegenPRP-Kit, Plateltex and one manual procedure. Vox Sang. 2009;97:110-118.

25. McCarrel T, Fortier L. Temporal growth factor release from platelet-rich plasma, trehalose lyophilized platelets, and bone marrow aspirate and their effect on tendon and ligament gene expression. J Orthop Res. 2009;27:1033-1042.

26. Phipps MC, Xu Y, Bellis SL. Delivery of platelet-derived growth factor as a chemotactic factor for mesenchymal stem cells by bone-mimetic electrospun scaffolds. PLoS One. 2012;7:e40831.

27. Randelli P, Arrigoni P, Ragone V, Aliprandi A, Cabitza P. Platelet rich plasma in arthroscopic rotator cuff repair: a prospective RCT study, 2-year follow-up. J Shoulder Elbow Surg. 2011;20:518-528.
28. Rodeo SA, Delos D, Weber A, Ju X, Cunningham ME, Fortier L, Maher S. What's new in orthopaedic research. J Bone Joint Surg Am. 2010;92:2491-2501.

29. Sanchez M, Anitua E, Azofra J, Andia I, Padilla S, Mujika I. Comparison of surgically repaired Achilles tendon tears using platelet-rich fibrin matrices. Am J Sports Med. 2007;35:245-251.

30. Schnabel LV, Lynch ME, van der Meulen MC, Yeager AE, Kornatowski MA, Nixon AJ. Mesenchymal stem cells and insulin-like growth factor-I gene-enhanced mesenchymal stem cells improve structural aspects of healing in equine flexor digitorum superficialis tendons. J Orthop Res. 2009;27:1392-1398.

31. Sundman EA, Cole BJ, Fortier LA. Growth factor and catabolic cytokine concentrations are influenced by the cellular composition of platelet-rich plasma. Am J Sports Med. 2011;39:21352140.

32. Tsay RC, Vo J, Burke A, Eisig SB, Lu HH, Landesberg R. Differential growth factor retention by platelet-rich plasma composites. J Oral Maxillofac Surg. 2005;63:521-528.

33. Visser LC, Arnoczky SP, Caballero O, Egerbacher M. Plateletrich fibrin constructs elute higher concentrations of transforming growth factor-betal and increase tendon cell proliferation over time when compared to blood clots: a comparative in vitro analysis. Vet Surg. 2010;39:811-817.

34. Volkmann I, Kumarswamy R, Pfaff N, Fiedler J, Dangwal S, Holzmann A, Batkai S, Geffers R, Lother A, Hein L, Thum T. MicroRNA-mediated epigenetic silencing of sirtuin 1 contributes to impaired angiogenic responses. Circ Res. 2013;113:997-1003.

35. Weibrich G, Kleis WK, Hafner G, Hitzler WE. Growth factor levels in platelet-rich plasma and correlations with donor age, sex, and platelet count. J Craniomaxillofac Surg. 2002;30:97-102.

36. Zhang F, Tsai S, Kato K, Yamanouchi D, Wang C, Rafii S, Liu B, Kent KC. Transforming growth factor-beta promotes recruitment of bone marrow cells and bone marrow-derived mesenchymal stem cells through stimulation of MCP-1 production in vascular smooth muscle cells. J Biol Chem. 2009;284:17564-17574.

37. Zumstein MA, Berger S, Schober M, Boileau P, Nyffeler RW, Horn M, Dahinden CA. Leukocyte- and platelet-rich fibrin (LPRF) for long-term delivery of growth factor in rotator cuff repair: review, preliminary results and future directions. Curr Pharm Biotechnol. 2012;13:1196-1206.

38. Zumstein MA, Rumian A, Lesbats V, Schaer M, Boileau P. Increased vascularization during early healing after biologic augmentation in repair of chronic rotator cuff tears using autologous leukocyte- and platelet-rich fibrin (L-PRF): a prospective randomized controlled pilot trial. J Shoulder Elbow Surg. 2014;23:3-12. 